\title{
The Optimization Design of Missile Maintenance Process Based on PERT Analysis
}

\author{
Wei Chen ${ }^{1,}$, , Di Zhang ${ }^{1}$, Chao Ma ${ }^{1}$ and Zhenzhou Bai ${ }^{1}$ \\ ${ }^{1}$ Air Force Logistics Academy, Xuzhou Jiangsu, 221000, China \\ ${ }^{2} 95937$ Troops, Fuxin Liaoning, 123000, China \\ ${ }^{*}$ Corresponding author
}

\begin{abstract}
The article adopted the PERT simulation analysis to optimize the maintenance procedure of the missile based on the network model. Through applying the PERT analysis, to analyze the network flow, with time as the reference standard of the optimal path, in front of modeling of projectile modeling difficult point analysis, can make us understand the problem should be pay attention to when modeling, then take corresponding measures to solve the problem.
\end{abstract}

\section{Keywords-PERT; missile maintenance; optimization design}

\section{INTRODUCTION}

During the process of Missile technology preparing, because of the limitation of human resources, the existing weapons and equipment, resulting in the technical preparation time is too long, often can't meet the needs of emergency preparations for the war, need to have a reasonable optimization command process and distribution equipment, such as technical preparation conditions ${ }^{[1-2]}$. PERT analysis based on the network analysis and time, the project planning and project control process are described as a whole, people can intuitively understand the inner link of each subsystem, increase the efficiency and better to the solve the problem.

\section{PERT ANALYSIS METHOD}

PERT simulation based on the monte carlo simulation, the main idea is to simulate the actual probability on the computer, the distribution of the computer to produce a given subject random work time, each have each work of random duration, is equivalent to project running time ${ }^{[3]}$. Every simulation, period and critical route may be different. After several simulation and statistical treatment, the key degree and the probability distribution of the work are obtained. PERT simulation can better reflect the working time of statistical regularity, can effective customer service classic PERT critical path and uniqueness assumption and the deficiency of traditional three estimates the identified critical path reliability is high, so as to improve the reliability of the plan.

\section{The BAsic IdeAs of Design Solution}

The longest line on the network map is the key route, and the work on the key route is called the critical work, and the common method of identifying critical routes is:

1. Take the longest route, which is the key line, by definition;
2. Calculating the time difference between work and work is equal to zero for critical work;

3. Calculating the mission probability of tasks that are completed in the network diagram at the time of the given task.

To calculate working hours, PERT estimates the time of the following three situations:

1. The most optimistic time: it means the time it takes to complete a job on a smooth basis, usually with the symbol alpha.

2. The most likely time: the time required to complete a job under normal working conditions, usually represented by the symbol beta.

3. The most pessimistic time: the time required to complete a job at the worst possible time, usually represented by the symbol gamma.

Set the time parameter and the working time parameter. The time parameters of the event include the earliest times and the latest events. The earliest time ( $\mathrm{j}$ ) of the item $\mathrm{j}$ is that it is the earliest possible time to start work. So, the earliest time of event $j$ should be equal to the sum of the hours worked on the longest line from the starting point to the item. The initial item number is 1 , and then there is

$$
\left\{\begin{array}{c}
t_{E}(1)=0 \\
t_{E}(j)=\max _{i}\left\{t_{E}(i)+D(i, j)\right\}
\end{array}\right.
$$

The latest delay in the event is called $t_{L}(i)$, indicating that it must begin at the latest in the condition that the task is not affected by the total duration of the task. Obviously, the earliest time of the final item is equal to the last time, which is equal to the total project period. Matters, then i should be equal to the time at the latest from the finish items on the shortest route to the items, items at the latest time and working hours, the smallest value of the difference between the result

$$
\begin{gathered}
t_{L}(n)=t_{E}(n) \\
t_{L}(i)=\min _{i}\left\{t_{L}(j)-D(i, j)\right\}
\end{gathered}
$$


The working time parameters include the earliest possible start time, the earliest possible completion time, the latest possible start time and the latest possible completion time.

Computational work time: the time difference between general including total time and free time, under the condition of not impact on the total time of task, a work start time can delay the biggest, total time, called the work record for $\mathrm{T}_{\mathrm{F}}(\mathrm{i}$, j), by definition:

$$
T_{F}(i, j)=t_{L F}(i, j)-t_{E F}(i, j)
$$

Some work (i, j) after the free time is to point to in does not affect the tight work under the condition of the earliest starting time, the starting time can delay the biggest, notes for the $F_{F}(i, j)$ is:

$$
F_{F}(i, j)=t_{E S}(i, j)-t_{E F}(i, j)
$$

Thus, the total time is the time to start construction within the scope of the overall work of change, free time is to work within the scope of this work nodes change of its starting time.

\section{The Optimization Model of Maintenance}

According to the principle of the simulation analysis of the working hours are assumed to be $\beta$ distribution, use Matlab to simulate, due to uncertain factors such as organizational management, equipment failure may affect technology to prepare project completed on schedule, with reference to the risk management theory, introducing work key and important degrees of two indicators for quantitative evaluation process each work position. From the point of view of ensuring that the project is completed on time, the critical work on key lines has the greatest impact on the period risk.

In a schedule, the key to a job doesn't mean that the job is much more likely to affect the risk of the project being completed. Therefore, besides to consider job key, also need to consider a job in the event of accident, influence on the whole project progress, can work by estimating the duration of the effect on the sensitivity of the project time limit for a project to evaluate:

$$
\begin{gathered}
\text { CRI }=\mathrm{q}(i, j)=\left|\operatorname{Corr}\left(D_{i j}, \mathrm{~T}\right)\right| \\
\left\{\begin{array}{c}
\operatorname{Corr}\left(D_{i j}, T\right)=\frac{\operatorname{Cov}\left(D_{i j}, T\right)}{\sigma\left(D_{i j}\right) \sigma(T)} \\
\operatorname{Cov}\left(D_{i j}, T\right)=E\left[\left(D_{i j}-E\left(D_{i j}\right)(T-E(T))\right)\right]
\end{array}\right.
\end{gathered}
$$

The upper formula is usually difficult to solve, and the formula can be obtained by simulation:

$$
\left\{\begin{array}{c}
\operatorname{Cov}\left(D_{i j}, T\right)=\frac{1}{N} \sum_{k=1}^{N}\left(D_{i-j}^{(k)}-E\left(D_{i-j}\right)\right) \cdot\left(T^{(k)}-E(T)\right) \\
E\left(D_{i j}\right)=\frac{1}{N} \sum_{k=1}^{N} D_{i j}^{(k)} \\
E(T)=\frac{1}{N} \sum_{k=1}^{N} T^{(k)} \\
\sigma\left(D_{i j}\right)=\sqrt{\frac{1}{N-1} \sum_{k=1}^{N}\left(D_{i j}^{(k)}-E\left(D_{i j}\right)\right)^{2}} \\
\sigma(T)=\sqrt{\frac{1}{N-1} K \sum_{k=1}^{N}\left(T^{(k)}-E(T)\right)^{2}}
\end{array}\right.
$$

$q(i, j) \in[0,1] . D_{i j}$ and $D_{i j}{ }^{(k)}$ duration of work (i, j) duration and duration of $\mathrm{k}$ simulations; $\mathrm{T}$ and $\mathrm{T}^{(\mathrm{K})}$ are the total period of work during the period of total construction and K.E (Dij) and $\sigma($ Dij) are the expected value and standard deviation of the continuous time of the work $(i, j)$ over $\mathrm{N}$ simulations. $\operatorname{Cov}\left(D_{i j}, T\right)$ and Corr $\left(D_{i j}, T\right)$ are the covariance and linearly dependent coefficients of the duration of work (i,j), and the linear correlation coefficients.

\section{The Maintenance Procedure Based on PERT}

Mapped the ammunition security according to the principle of PERT analysis method, the concrete process of forming a more comprehensive network diagram, analyze network diagram, missile technology security refers to the missile by storage condition after decomposition, testing, assembly and a series of operation into the level of combat readiness state (or other combat readiness state) process, involving a number of equipment, tools, and personnel, through the process analysis, build a network model, the basic idea is shown in Fig 1.

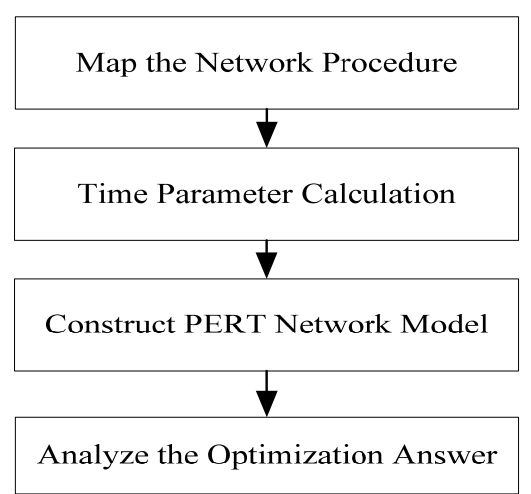

FIGUREI. MISSILE TECHNOLOGY SUPPORT BASED ON PERT NETWORK MODEL

Network model is established, by analyzing the situation of each link may appear, to simulate the working time, 
because the work environment, personnel has uncertainties, there are many uncertainty factors in the process of execution, such as equipment failure need to temporarily enable backup pieces, insufficient number of personnel or operation is not enough skilled work delay, etc., thus the safeguard link safeguard time will fluctuate within a certain range. By establishing the model of missile technology security based on PERT network model, each process will be according to the equipment, time, human resources demand in the network diagram, said at the same time, through the arrow lines show the logic relation of work, the working time for simulating the most optimistic time, three time and perhaps the most pessimistic time, combined with the time of equipment, manpower needs to establish the relationship between function and construction of complete process of PERT network model.

\section{CONCLUSION}

In practice, the influence of various parameters such as equipment, time, human resources and other parameters on the process protection. Especially for missile the maintenance time, maintenance content is various complex work, the distribution of personnel is particularly important, in the number of certain situations, tasks through PERT analysis reasonable arrangement of personnel for operation, is neither a waste of resources, and can make the completion of work safety.

\section{REFERENCE}

[1] Zuo Chuanyou. The technical preparation process for missile technology of PERT method [J]. Journal of naval aviation engineering college, 2008.23 (2) : 34-38.

[2] Guo Xiaowei, Ma Dengwu, Deng Li. Aviation ammunition based on PERT network security personnel optimization configuration [M]. Journal of Beijing university of aeronautics and astronautics, 2014.40 (1) : 69-74.

[3] Zhang Wei. The sensitivity analysis of critical programs in the Internet in the Internet. Technology economy, 2003, 28 (12) : 114-118 